\title{
Anomalous Thermal Transport in Quantum Wires
}

\author{
Rosario Fazio $^{(1)}$, F.W.J. Hekking ${ }^{(2,3)}$, and D.E. Khmelnitskii ${ }^{(2,4)}$ \\ (1) Istituto di Fisica, Universitá di Catania, EJ INFM, Viale A. Doria 6, I-95129 Catania, Italy \\ ${ }^{(2)}$ Cavendish Laboratory, University of Cambridge, Madingley Road, Cambridge CB3 OHE, United \\ Kingdom \\ (3) Theoretische Physik III, Ruhr-Universität Bochum, 44780 Bochum, Germany \\ ${ }^{(4)}$ L.D. Landau Institute for Theoretical Physics, 117940 Moscow, Russia
}

(August 20, 2019)

\begin{abstract}
We study thermal transport in a one-dimensional quantum wire, connected to reservoirs. Despite of the absence of electron backscattering, interactions in the wire strongly influence thermal transport. Electrons propagate with unitary transmission through the wire and electric conductance is not affected. Energy, however, is carried by bosonic excitations (plasmons) which suffer from scattering even on scales much larger than the Fermi wavelength. If the electron density varies randomly, plasmons are localized and charge-energy separation occurs. We also discuss the effect of plasmon-plasmon interaction using Levinson's theory of nonlocal heat transport.
\end{abstract}


In the Fermi liquid theory of thermoelectric transport, both charge and energy are carried by fermionic quasiparticlest. This manifests itself in a universal relation between the electric conductivity $\sigma$ and thermal conductivity $\kappa$, known as the Wiedemann-Franz (WF) law $\left(k_{B}=\hbar=1\right)$,

$$
\frac{\kappa}{\sigma T}=\frac{\pi^{2}}{3 e^{2}}
$$

The validity of Eq. (11) has been confirmed in the case of arbitrary impurity scattering $\mathrm{B}$ and in the presence of electron-electron interactions ${ }^{3}$ within the Fermi liquid approach.

At low temperatures, in conductors of small size, phase-coherent electron propagation dominates transport. Mesoscopic contributions to thermoelectric coefficients in the diffusive regime are quite significant 1 . The thermoelectric coefficients of a ballistic quantum point contact have been studied experimentally 5 and theoretically using a scattering approach 6 . In this case, the observed violations of the WF law can be attributed to the strong energy dependence of the scattering matrix.

Electron-electron interactions in low-dimensional systems may lead to non Fermi liquid behavior. In this context transport properties of quantum wires are of considerable current interest $\mathrm{\theta}$. Theoretically these systems are studied in the framework of the Luttinger liquid (LL) model 10 . Revived interest in the transport in LLs was triggered by the work of Kane and Fisher11. The effect of interaction on the electric conductance crucially depends on the way in which the quantum wire is connected to the measuring leads (reservoirs) 12 . More recently thermal transport in a LL was considered 13 and deviations from the Fermi liquid relation, Eq. (1), were predicted.

The low energy excitations of an interacting one-dimensional (1D) system are long wavelength density oscillations (plasmons) which have bosonic character. This has drastic consequences for thermoelectric transport, since the scattering properties of the electrons (which determine the transfer of charge) are in general quite different from the scattering properties of the plasmons (responsible for the transfer of energy). In this paper we study this distinct difference by considering situations in which the dc electric conductance is not affected by interaction in contrast to the thermal conductance. Specifically we consider a one channel LL with spatially varying density, which is connected to two reservoirs as it is shown in Fig. 1. If the spatial variation related to these inhomogeneities occurs on a length scale much larger than the Fermi wavelength $\lambda_{F}$, electrons will not suffer any backscattering. The electric DC conductance will therefore be given by the universal value $G=e^{2} / 2 \pi$. However, plasmons with wavelengths much larger than $\lambda_{F}$ will suffer backscattering. Under these circumstances the thermal conductance is strongly affected by interactions.

The Hamiltonian which describes such an inhomogeneous electron liquid can be written as $14=\int d x \mathcal{H}(x)$, where

$$
\mathcal{H}(x)=\frac{p^{2}}{2 m n(x)}+\frac{1}{2}\left[V_{0}+\frac{\pi^{2}}{m} n(x)\right]\left(\partial_{x} n u\right)^{2} .
$$

Here, $u(x)$ and $p(x)$ are the displacement of the electron liquid and the conjugate momentum, respectively, satisfying $\left[u(x), p\left(x^{\prime}\right)\right]=i \delta\left(x-x^{\prime}\right)$. The local average electron concentration is $n(x)$. Furthermore, $V_{0}$ is the interaction strength (the zero-momentum Fourier component of the interaction potential). The connection of a quantum wire of length $d$ to reservoirs 
is modeled by a space-dependent interaction $V_{0}(x)\left(V_{0}(x)=V_{0}\right.$ if $0<x<d$ and zero otherwise) 2, 2 . For later use we define the parameter $g=\left(1+V_{0} / \pi v_{F}\right)^{-1 / 2}$, characterizing the interaction strength in the wire. We furthermore take $n(x)=n_{0} \equiv m v_{F} / \pi$ in the reservoirs $\left(i . e\right.$. for $x<0$ or $x>d$. Using the continuity equation $\partial_{x} J_{E}(x)+\partial_{t} \mathcal{H}(x)=0$, the energy current $J_{E}(x)$ can be expressed in terms of $u(x)$ and $p(x)$,

$$
J_{E}(x)=-\frac{1}{2 m}\left[V_{0}+\frac{\pi^{2}}{m} n(x)\right]\left\{p(x), \partial_{x}(n u)\right\},
$$

where $\{\ldots, \ldots\}$ denotes the anticommutator. We will show that the calculation of the average energy current amounts to the solution of a well defined scattering problem in analogy to the well known Landauer-Büttiker approach for quantum transport in noninteracting electron systems 16 .

To this end, we diagonalize the Hamiltonian (2) using a basis of scattering states $\phi_{k, \alpha}(x)$ which satisfy the wave equation

$$
-\hat{h} \phi_{k, \alpha}=\omega_{k}^{2} \phi_{k, \alpha}(x)
$$

where $\omega_{k}$ is the energy of the given mode and

$$
\hat{h}=\sqrt{n(x)} \partial_{x}\left(V_{0} / m+\pi^{2} n(x) / m^{2}\right) \partial_{x} \sqrt{n(x)} .
$$

The index $\alpha=r, l$ labels the states incident from the right and left reservoir, respectively, with wavevector $k$. The scattering states have asymptotics

$$
\begin{aligned}
\phi_{k, l}(x) & =e^{i k x}+r_{\omega_{k}} e^{-i k x} \text { for } x \rightarrow-\infty, \\
& =t_{\omega_{k}} e^{i k x} \text { for } x \rightarrow \infty ; \\
\phi_{k, r}(x) & =t_{\omega_{k}} e^{-i k x} \text { for } x \rightarrow-\infty, \\
& =e^{-i k x}-r_{\omega_{k}}^{*}\left(t_{\omega_{k}} / t_{\omega_{k}}^{*}\right) e^{i k x} \text { for } x \rightarrow \infty,
\end{aligned}
$$

where $r_{\omega_{k}}$ and $t_{\omega_{k}}$ are the reflection and transmission amplitude, respectively. We emphasize that $\phi_{k, \alpha}$ describe plasmon waves rather than electronic excitations. The diagonalized Hamiltonian is given by

$$
H=(1 / 2) \sum_{\alpha} \int d k \omega_{k}\left(b_{k, \alpha}^{\dagger} b_{k, \alpha}+b_{k, \alpha} b_{k, \alpha}^{\dagger}\right)
$$

Here, the operators $b$ and $b^{\dagger}$ obey the Bose commutation relation $\left[b_{k, \alpha}, b_{k^{\prime}, \alpha^{\prime}}^{\dagger}\right]=\delta_{\alpha, \alpha^{\prime}} \delta\left(k-k^{\prime}\right)$. The displacement field and its conjugate momentum can be expressed as

$$
\begin{gathered}
p(x)=\sum_{\alpha} \int_{0}^{\infty} d k \frac{1}{i} \sqrt{\frac{\Omega_{k}(x)}{2}}\left[\phi_{k, \alpha}(x) b_{k, \alpha}-\phi_{k, \alpha}^{*}(x) b_{k, \alpha}^{\dagger}\right], \\
u(x)=\sum_{\alpha} \int_{0}^{\infty} d k \sqrt{\frac{1}{2 \Omega_{k}(x)}}\left[\phi_{k, \alpha}(x) b_{k, \alpha}+\phi_{k, \alpha}^{*}(x) b_{k, \alpha}^{\dagger}\right],
\end{gathered}
$$

where $\Omega_{k}(x)=m n(x) \omega_{k}$. At this point we want to stress that the above formulation is applicable because each mode contributes independently to the energy flux. A similar 
approach cannot be applied to determine the electric current, as the charge is transported through the sample via a complicated superposition of plasmon modes.

Now we are in a position to calculate the average energy current. This quantity is spaceindependent because of current conservation, hence we calculate it, say, for $x \rightarrow \infty$. We substitute the appropriate asymptotics from (5) into (7), then perform the Gibbs average of the anticommutator in (3) with respect to the Hamiltonian (6). As a result we find

$$
\left\langle J_{E}\right\rangle=\frac{1}{2 \pi} \int_{0}^{\infty} d \omega\left|t_{\omega}\right|^{2}\left[n_{l}(\omega)-n_{r}(\omega)\right],
$$

where $n_{\alpha}(\omega)$ is the Bose function of reservoir $\alpha$. This result holds as long as electrons are not backscattered by inhomogeneities. In the linear response regime, when the temperature difference $\Delta T$ between the reservoirs is vanishingly small, the thermal conductance is readily evaluated to be

$$
K=\frac{1}{8 \pi T^{2}} \int_{0}^{\infty} d \omega \frac{\omega^{2}}{\sinh ^{2}(\beta \omega / 2)}\left|t_{\omega}\right|^{2},
$$

where $\beta$ is the inverse temperature $1 / T$. For noninteracting electrons, $\left|t_{\omega}\right|=1$, this expression reduces to the well-known result $K=\pi T / 6$. Eq. (9) shows that inhomogeneities strongly affect thermal transport in an interacting quantum wire, even in the absence of any electron backscattering. This is essentially the analogue of the Kapitza boundary resistance 17. Below we study the behavior of $K$, Eq. (9), in two relevant limits.

(i) The simplest situation occurs when an interacting wire of finite length with a constant electron density $n_{0}$ is connected adiabatically to two noninteracting reservoirs. In this case the solutions of Eq. (4) inside the wire are plane waves, with momentum $g k$. The plasmon transmission coefficient can be calculated explicitly; it is strongly frequency-dependent with characteristic frequency $v_{F} / g d$ due to the mismatch of momenta at $x=0$ and $x=d$. As a result, $K$ will be suppressed below its noninteracting value. The Lorentz number $L=K / G T=2 \pi K / e^{2} T$ as a function of temperature and interaction strength is plotted in Fig. 目. In the low-temperature limit, the Lorentz number attains its noninteracting value $L_{0}=\pi^{2} /\left(3 e^{2}\right)$ (since the zero frequency transmission coefficient equals unity) and decreases with increasing temperature and interaction strength. Notwithstanding the fact that a decrease of $L$ is a genuine feature of the system, the actual quantitative suppression depends on the specific choice of the space dependence of the interaction strength. Note, finally, the profound difference between our results and those obtained in 13 . For an infinitely long interacting wire without reservoirs, the electric conductance is renormalized 11 . However, the thermal conductance is unaffected by interactions and an enhanced Lorentz number signals the breakdown of Fermi liquid theory 13 . In the presence of reservoirs, the electric conductance is unrenormalized 22 but the thermal conductance is suppressed, leading to a suppression of $L$.

(ii) Even in the best samples, random variations of electron density on scales $l_{D}$ much larger than $\lambda_{F}$ are unavoidable. Plasmons, therefore, propagate in a random medium and, depending on their energy, can be localized. We anticipate that in some temperature range charge and energy are spatially separated (charge-energy separation). In order to model the 
randomness, we decompose the electron density as $n(x)=n_{0}+\delta n(x)$ where the random component has a normal distribution with variance

$$
\langle\delta n(x) \delta n(y)\rangle=n_{0}^{2} \delta\left(\frac{x-y}{l_{D}}\right) .
$$

We calculated the average plasmon transmission coefficient with the help of the invariant embedding technique developed in Ref.18. The decay of the plasmon wave inside the interacting wire is governed by the lengthscale

$$
\xi_{\omega}=\frac{v_{F}}{g} \tau_{D}(\omega)=\frac{2 \pi^{2}}{g^{6}} \frac{v_{F}^{2}}{V_{0}^{2}} \frac{v_{F}^{2}}{l_{D} \omega^{2}},
$$

where we introduced the lifetime for impurity scattering $\tau_{D}(\omega)$. Eq. (10) was derived using the Golden Rule in Ref.14. The transmission coefficient asymptotically decays as

$$
\left|t_{\omega}\right|^{2} \sim e^{-d / \xi_{\omega}}
$$

for $d \gg \xi_{\omega}$. At low enough temperatures, $T<\omega_{1}^{*}=\left(2 \pi^{2} v_{F}^{4} / g^{6} l_{D} V_{0}^{2} d\right)^{1 / 2}$, the thermal plasmons propagate ballistically. In the opposite limit $T>\omega_{1}^{*}$, localization of high-frequency plasmons occurs and the thermal conductance rapidly saturates to some constant value $K_{0}$ as sketched in Fig. 3.

A finite plasmon lifetime is not only caused by scattering off inhomogeneities. The nonzero curvature of the single electron spectrum leads to interactions between plasmons. The effect of these two scattering mechanisms on the plasmon kinetics is very different, as we will discuss in the remainder of this paper. Scattering off inhomogeneities relaxes the momentum but does not lead to thermal equilibrium. This is established by plasmon interactions which, in lowest order, are described by the cubic Hamiltonian 10

$$
H_{\mathrm{int}}=-\frac{1}{2} \int d x \frac{p^{2}}{m n(x)} \partial_{x}(n u)-\frac{\pi^{2}}{6 m} \int d x\left(\partial_{x} n_{0} u\right)^{3} .
$$

The rate for three-plasmon scattering can be calculated using the Golden Rule. Special care is needed, however, in this case. Since the dispersion relation is linear in $k$, momentum and energy conservation are simultaneously satisfied, hence the rate is infinite. In the presence of impurity scattering it can be regularized, because a state with given energy $\omega$ corresponds to a wave packet with $\langle k\rangle=g \omega / v_{F}$ and a width $\left\langle(\delta k)^{2}\right\rangle \sim\left(g / v_{F} \tau_{D}(\omega)\right)^{2}$. Using the broadened dispersion $\omega_{k}=v_{F}|k| / g+i \tau_{D}^{-1}\left(v_{F} k / g\right)$, we find the rate associated with spontaneous decay of a plasmon

$$
\frac{1}{\tau(\omega)}=\frac{\left(3+g^{2}\right)}{16 g^{2}} \frac{\omega^{2}}{n_{0}^{2} V_{0}^{2}} \frac{v_{F}}{l_{D}} .
$$

Interactions are important for plasmons with energies larger than the cross-over frequency $\omega_{2}^{*} \sim v_{F} / \sqrt{d \lambda_{F}}$, since they experience interactions while diffusing over distances of the order of the length of the wire $d$. The ratio $\left(\omega_{2}^{*} / \omega_{1}^{*}\right)^{2} \sim\left(V_{0} / v_{F}\right)^{2} l_{D} / \lambda_{F}$ should be much larger than unity. This, together with the condition $\left\langle(\delta k)^{2}\right\rangle \ll\langle k\rangle^{2}$ for the wave packet discussed above, implies that $\left(\lambda_{F} / l_{D}\right)^{1 / 2} \ll V_{0} / v_{F} \ll 1$. 
The scattering approach of thermal transport, resulting in Eq. (8), applies when both reservoirs are kept at a temperature smaller than $\omega_{2}^{*}$. At temperatures larger than $\omega_{2}^{*}$ local equilibrium tends to be established. The difference between these two regimes can be distinguished, e.g., by considering the nonlinear response to a large temperature difference $\Delta T$ between the reservoirs. Using the scattering approach Eq. (8) one finds

$$
J_{E} \sim \Delta T \text { if } \Delta T<\omega_{2}^{*} .
$$

If $\Delta T \gg \omega_{2}^{*}$, interaction between plasmons can no longer be ignored. Using Levinson's theory 19 of phonon heat transport in disordered dielectrics and semiconductors, we will show that far from equilibrium the energy current depends algebraically on the temperature difference $\Delta T$.

Below we closely follow the derivation given in Ref.19. Since low energy plasmons propagate easier through a disordered wire than high energy ones, the plasmon distribution function is depleted at energies smaller than a certain cross-over frequency $\bar{\omega}$. Therefore, in the presence of plasmon interactions, down energy conversion occurs from thermal to low energy plasmons. The latter then carry the energy to the cold end of the wire. The relevant time scale for down conversion is

$$
\frac{1}{\tau_{B}(\omega)}=\frac{2}{\tau(T)} \frac{\omega}{T}=\frac{\left(3+g^{2}\right)}{8 g^{2}} \frac{\omega T}{n_{0}^{2} V_{0}^{2}} \frac{v_{F}}{l_{D}} .
$$

Comparing $\tau_{B}$ with the diffusion time $g d^{2} / v_{F} \xi_{\omega}$ one finds

$$
\bar{\omega}=T\left(\frac{d}{L_{T}}\right)^{-2 / 3}
$$

where $L_{T}=\left\{4 \pi n_{0} v_{F}^{2} /\left[g^{5}\left(3+g^{2}\right)\right]^{1 / 2}\right\} T^{-2}$. For frequencies below $\bar{\omega}$ the depleted distribution function obeys the stationary diffusion equation $\left(v_{F} \xi_{\omega} / g\right) \partial_{x}^{2} n=1 / \tau(T)$, hence it is given by

$$
n(\omega) \sim\left(\frac{d}{L_{T}}\right)^{2}\left(\frac{\omega}{T}\right)^{2} .
$$

For frequencies larger than $\bar{\omega}$ the thermal distribution $n(\omega) \sim T / \omega$ is recovered. Using this distribution function it is possible to evaluate the nonlinear energy current $J_{E} \sim$ $\int d \omega \omega \xi_{\omega} n(\omega) / d$

$$
J_{E} \sim(\Delta T)^{4 / 3} \text { if } \Delta T>\omega_{2}^{*} .
$$

The power law given in Eq. (17) is universal: it does not depend on the interaction strength. Still, this algebraic behavior is a genuine effect of the electron-electron interaction: in the noninteracting case, plasmons are ballistic at all frequencies and Eq. (13) applies. The nonlinearity in Eq. (17) is a result of the interplay between electron-electron interactions and plasmon-plasmon interactions, which provides a mechanism for redistribution of energy over the plasmon spectrum.

Acknowledgments We thank Ya.M. Blanter, K. Samokhin and especially E. Paladino for valuable discussions. We acknowledge the financial support of INFM (PRA-QTMD) and the European Community (Contract ERB-CHBI-CT941764). 


\section{REFERENCES}

${ }^{1}$ A.A. Abrikosov, Fundamentals of the theory of metals, North-Holland (Amsterdam) 1988.

${ }^{2}$ G.V. Chester and A. Thellung, Proc. Phys. Soc. London 77, 1005 (1961).

${ }^{3}$ C. Castellani et al., Phys. Rev. Lett. 59, 477 (1987).

${ }^{4}$ A.V. Anisovich et al., Pis'ma Zh. Eksp. Teor. Fiz. 45, 237 (1987) [JETP Lett. 45, 295 (1987)]; G.B. Lesovik and D.E. Khmel'nitskii, Zh. Eksp. Teor. Fiz. 94, 164 (1988) [Sov. Phys. JETP 67, 957 (1988)].

${ }^{5}$ L.W. Molenkamp et al., Phys. Rev. Lett 65, 1052 (1990); A. Dzurak et al., J. Phys. Condens. Matter 5, 8055 (1993).

${ }^{6}$ U. Sivan and Y. Imry, Phys. Rev. B 33, 551 (1986).

${ }^{7}$ P.N. Butcher, J. Phys. Condens. Matter 2, 4869 (1990).

${ }^{8}$ H. van Houten et al., Semicond. Sci. Technol. 7, B215 (1992).

${ }^{9}$ S. Tarucha et al., Sol. State Comm. 94, 413 (1995); A. Yacoby et al., Phys. Rev. Lett. 76, 4612 (1996).

${ }^{10}$ F.D.M. Haldane, J. Phys. C 14, 2585 (1981).

${ }^{11}$ C.L. Kane and M.P.A. Fisher, Phys. Rev. Lett. 68, 1220 (1992).

12 D. L. Maslov and M. Stone, Phys. Rev. B 52, R5539 (1995); V. V. Ponomarenko, ibid 52, R8666 (1995); I. Safi and H. J. Schulz, ibid 52, R17040 (1995).

${ }^{13}$ C.L. Kane and M.P.A. Fisher, Phys. Rev. Lett. 763192 (1996).

${ }^{14}$ A. Gramada and M.E. Raikh, Phys. Rev. B 55, 7673 (1997).

${ }^{15}$ A description of reservoirs in terms of noninteracting electrons is appropriate as long as time-dependent fields are absent, see Ya. Blanter et al., cond-mat/9710299 (unpublished).

16 See M. Büttiker, Phys. Rev. B 46, 12485 (1992), and references therein.

17 See, e.g., E.T. Swartz and R.O. Pohl, Rev. Mod. Phys. 61, 605 (1989).

${ }^{18}$ R. Rammal and B. Doucot, J. Physique 48, 509 (1987).

${ }^{19}$ Y.B. Levinson, Sol. State Comm. 36, 73 (1980).

${ }^{20}$ Y.B. Levinson, in Nonequilibrium Phonons in Nonmetallic Crystals, edited by W. Eisenmenger and A.A. Kaplyanskii, North-Holland, Amsterdam, 1986, page 91. 


\section{FIGURES}

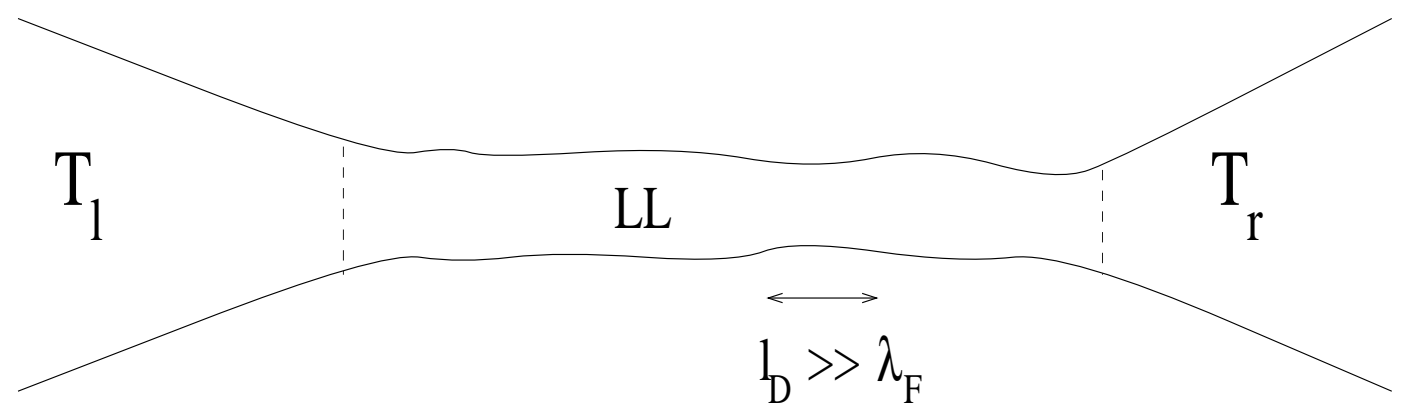

FIG. 1. The 1D wire, connected adiabatically to two reservoirs kept at different temperatures. The wire has some inhomogeneities on a scale which is much larger than $\lambda_{F}$.

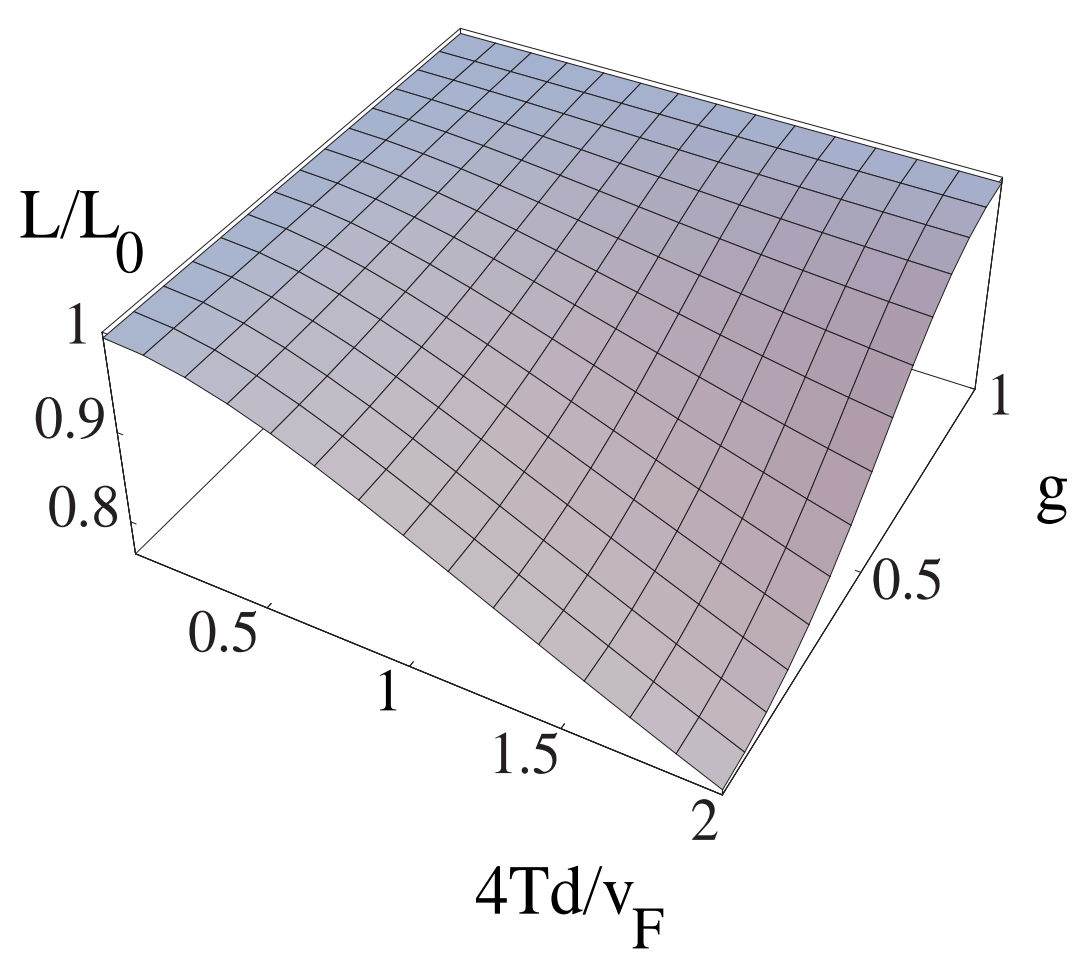

FIG. 2. The Lorentz number for an ideal wire attached to reservoirs is plotted as a function of the temperature and the interaction strength. 


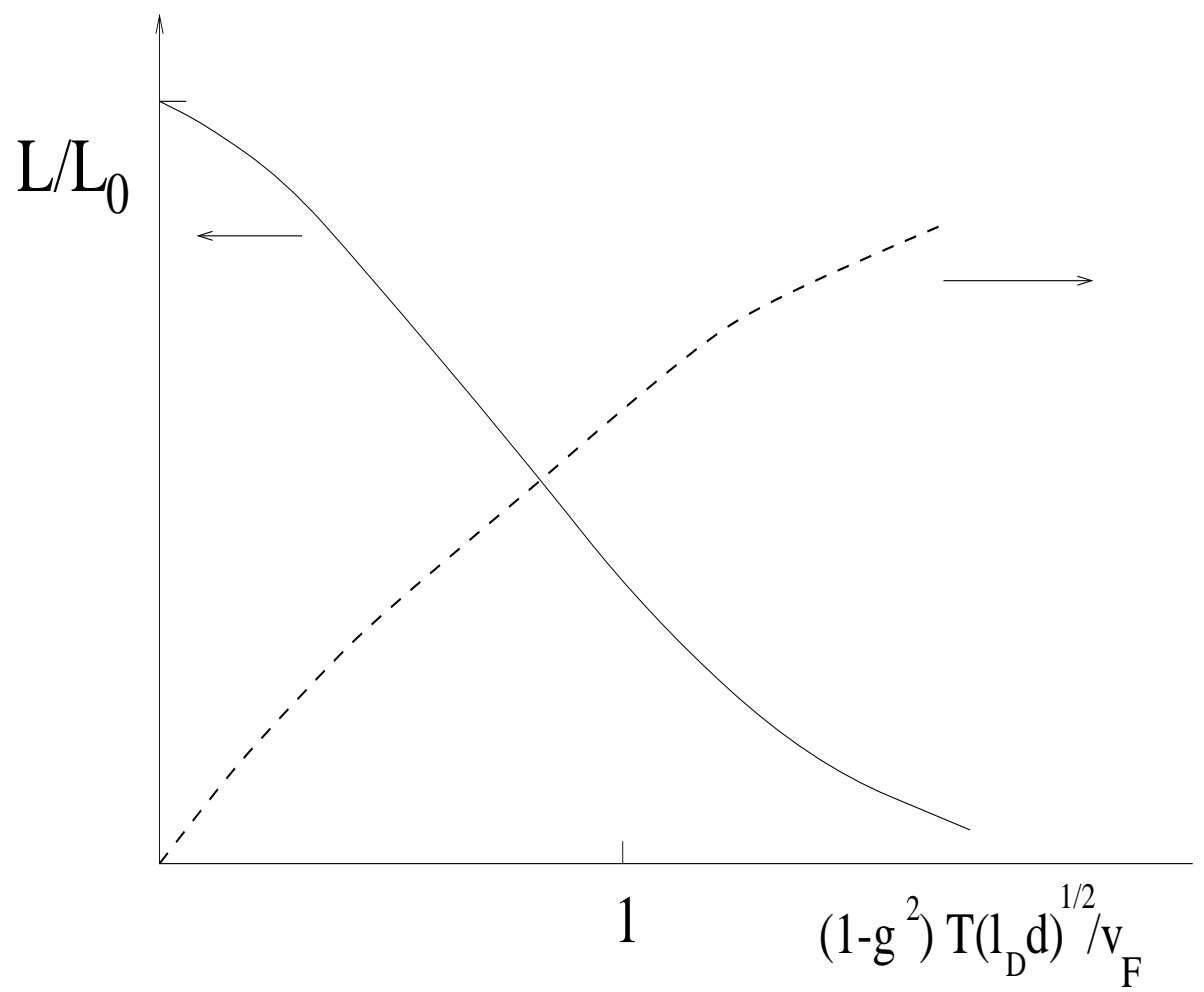

FIG. 3. The behavior of the Lorentz number and the thermal conductance is sketched for the case in which the wire is disordered. The saturation of $K$ at some value $K_{0}$ is related to the localization of the high frequency plasmons. 